\title{
Kardia Mobile applicability in clinical practice: A comparison of Kardia Mobile and standard 12-lead electrocardiogram records in 100 consecutive patients of a tertiary cardiovascular care center
}

\author{
Lukasz Koltowski, Pawel Balsam, Renata Glowczynska, Jakub K. Rokicki, \\ Michal Peller, Jakub Maksym, Leszek Blicharz, Kacper Maciejewski, \\ Magdalena Niedziela, Grzegorz Opolski, Marcin Grabowski \\ $1^{\text {st }}$ Chair and Department of Cardiology, Medical University of Warsaw, Poland
}

\begin{abstract}
Background: Mobile devices are gaining a rising number of users in all countries around the globe. Novel solutions to diagnose patients with out-of-hospital onset of arrhythmic symptoms can be easily used to record such events, but the effectiveness of these devices remain unknown.

Methods: In a group of 100 consecutive patients of an academic cardiology care center (mean age $68 \pm 14.2$ years, males: 66\%) a standard 12-lead electrocardiogram (ECG) and a Kardia Mobile (KM) record were registered. Both versions were assessed by three independant groups of physicians.

Results: The analysis of comparisons for standard ECG and KM records showed that the latter is of lower quality $(p<0.001)$. It was non-inferior for detection of atrial fibrillation and atrial flutter, showed weaker rhythm detection in pacemaker stimulation $(p=0.008)$, and was superior in sinus rhythm detection $(p=0.02)$, though. The sensitivity of KM to detect pathological Q-wave was low compared to specificity (20.6\% vs. 93.7\%, respectively, $p<0.001$ ). Basic intervals measured by the KM device, namely $P Q, R R$, and $Q T$ were significantly different (shorter) than those observed in the standard ECG method (160 ms vs. $180 \mathrm{~ms}[p<0.001], 853 \mathrm{~ms} v \mathrm{~s} .880 \mathrm{~ms}[\mathrm{p}=0.03]$ and $393 \mathrm{~ms} v \mathrm{~s} .400 \mathrm{~ms}$ $[p<0.001]$, respectively).

Conclusions: Initial and indicative value of atrial fibrillation and atrial flutter detection in KM is comparable to results achieved in standard ECG. KM was superior in detection of sinus rhythm than eye-ball evaluation of 12-lead ECG. Though, the PQ and QT intervals were shorter in KM as compared to 12-lead ECG. Clinical value needs to be verified in large studies, though. (Cardiol J 2021; 28, 4: 543-548)
\end{abstract}

Key words: arrhythmia, telemedicine, mobile, electrocardiogram, atrial fibrillation

\section{Introduction}

Increasing versatility of mobile devices provides an opportunity to implement them in the diagnosis and follow-up of patients with cardiovascular disorders. Kardia Mobile (KM) (AliveCor Inc., San Francisco, CA, USA) is a portable, mo- bile, connected electrocardiogram (ECG) device available to iOS and Android platform smartphone owners. It consists of a small device with two conducting plates that wirelessly connect with a smartphone, and an application installed on user smartphones. It enables one-lead ECG recording e.g. in cases of the onset of unsettling symptoms

Address for correspondence: Paweł Balsam, MD, PhD, $1^{\text {st }}$ Chair and Department of Cardiology, Medical University of Warsaw, ul. Banacha 1a, 02-097 Warszawa, Poland, tel: +48 2259919 58, fax: +48 2259919 57, e-mail: pawel.balsam@wum.edu.pl

Received: 12.06.2018 Accepted: 22.11.2018

This article is available in open access under Creative Common Attribution-Non-Commercial-No Derivatives 4.0 International (CC BY-NC-ND 4.0) license, allowing to download articles and share them with others as long as they credit the authors and the publisher, but without permission to change them in any way or use them commercially. 
(palpitations, chest pain, dyspnea, and others). KM was designed to detect periods of atrial fibrillation (AF), which, if confirmed by the Food and Drug Administration (FDA)-approved algorithm, can then be reported to the physician responsible for the follow-up of a given patient. The accessibility of KM has prompted the present evaluation of its usefulness in comparison with 12-lead ECG recordings in assessing the underlying rhythm and basic ECG parameters (PQ, RR, and QT intervals). The aim herein was to establish protocol, according to evidence-based medicine, on whether it has acceptable sensitivity and specificity, and thus, useful and reliable for medical professionals.

\section{Methods}

\section{Recruitment}

A total of 100 consecutive patients of mean age $68 \pm 14.2$, with male subjects constituting $66 \%$ of the group of a tertiary cardiovascular care center were included in the study. Baseline characteristics can be found in the Table 1 .

The patients were admitted to hospital elective diagnostic and treatment procedures for various cardiac conditions (arrhythmias, conduction disorders, stable coronary disease, hypertension and others). The group consisted of 100 patients consecutively admitted to the documented center between July $1^{\text {st }}$ and July $31^{\text {st }} 2015$. The inclusion criterium was undergoing regular 12-lead ECG due to standard diagnosis on admission in stable state. All patients who urgently needed medical care were excluded. Upon referral for a 12-lead ECG, each patient was asked to provide informed consent for additional KM ECG recording. All patients agreed to take part in the study. The subjects' clinical state was stable at the time of ECG recording. First a 12-lead ECG was performed and KM ECG was recorded directly after this procedure. Two technicians were responsible for 12-lead ECG measurements and one physician to record KM ECGs.

\section{ECG assessment}

Both 12-lead and KM ECG records were analyzed by three independent teams comprised of two cardiologists each. ECG interpretation was carried out according to a previously elaborated online form, consistent with the Polish ECG Description Guidelines [1], which required assessment of the following: ECG quality (good, acceptable, poor), rhythm (sinus rhythm, AF, atrial flutter [AFl] or pacemaker rhythm), presence of pathological $\mathrm{Q}$ wave as well as $\mathrm{PQ}, \mathrm{RR}$ and $\mathrm{QT}$ measurements.
Table 1. Patient characteristics.

\begin{tabular}{|c|c|}
\hline Baseline demographics & $\begin{array}{c}N(\%) \text { or } \\
\text { mean } \pm \text { SD }\end{array}$ \\
\hline Gender [male] & $66(66.0 \%)$ \\
\hline Age [years] & $68.0 \pm 14.2$ \\
\hline Body mass [kg] & $80.7 \pm 15.9$ \\
\hline Height $[\mathrm{m}]$ & $1.7 \pm 0.1$ \\
\hline Body mass index & $28.0 \pm 4.8$ \\
\hline \multicolumn{2}{|l|}{ Medical history } \\
\hline Nicotinism (history): & $40(43.5 \%)$ \\
\hline Nicotinism active & $9(9.8 \%)$ \\
\hline Packyears cumulative & $33.4 \pm 22.5$ \\
\hline Diabetes mellitus & $20(20.4 \%)$ \\
\hline Hypertension & $67(68.4 \%)$ \\
\hline Dyslipidemia & $45(46.4 \%)$ \\
\hline Chronic kidney disease: & $32(32.7 \%)$ \\
\hline CKD G2 & $2(2.0 \%)$ \\
\hline CKD G3 & $23(23.5 \%)$ \\
\hline CKD G4 & $7(7.1 \%)$ \\
\hline CKD G5 & $1(1.0 \%)$ \\
\hline Thyroid dysfunction: & $18(18.4 \%)$ \\
\hline Hypothyroidism & $9(9.18 \%)$ \\
\hline Hyperthyroidism & $9(9.18 \%)$ \\
\hline COPD: & $6(6.12 \%)$ \\
\hline Parkinson's disease & $1(1.02 \%)$ \\
\hline Cerebrovascular disease & $17(17.35 \%)$ \\
\hline Peripheral artery disease & $12(12.24 \%)$ \\
\hline \multicolumn{2}{|l|}{ Cardiovascular history } \\
\hline Stable angina (angina pectoris) & $46(47.4 \%)$ \\
\hline ACS (admission) & $15(15.31 \%)$ \\
\hline Myocardial infarction (history) & $25(25.5 \%)$ \\
\hline PCI/CABG (history) & $27(27.6 \%)$ \\
\hline Cardiac surgery (other than CABG) & $3(3.1 \%)$ \\
\hline Heart failure: & $43(43.9 \%)$ \\
\hline NYHA II & $28(28.6 \%)$ \\
\hline NYHA III & $9(9.2 \%)$ \\
\hline NYHA IV & $6(6.1 \%)$ \\
\hline LVEF [\%] & $49 \pm 14$ \\
\hline Atrial fibrillation: & $34(34.7 \%)$ \\
\hline Paroxysmal & $16(16.33 \%)$ \\
\hline Persistent & $4(4.1 \%)$ \\
\hline Chronic & $14(14.3 \%)$ \\
\hline CIED implanted & $34(34.7 \%)$ \\
\hline Pacemaker: & $24(24.5 \%)$ \\
\hline AAI & $2(2.0 \%)$ \\
\hline VVI & $7(7.1 \%)$ \\
\hline DDD & $15(15.3 \%)$ \\
\hline ICD & $5(5.1 \%)$ \\
\hline CRT & $5(5.1 \%)$ \\
\hline Ablation & $6(6.1 \%)$ \\
\hline
\end{tabular}

ACS - acute coronary syndrome at the time of admission; CABG coronary artery bypass grafting; CIED - cardiac implantable electronic device; CKD - chronic kidney disease (G2-G5 - CKD stages); COPD - chronic obstructive pulmonary disease; CRT cardiac resynchronization therapy device; ICD - implantable cardioverter-defibrillator; LVEF - left ventricular ejection fraction measured during the hospitalization; NYHA - New York Heart Association functional classification; $\mathrm{PCl}$ - percutaneous coronary intervention 
In order to avoid cognitive and measurement bias, all KM ECG records were printed out in 1:1 ratio and assessed in paper-based form. A database comprised of answers provided by the teams. The study protocol and informed consent form was approved by the local bioethics committee.

\section{Statistical analysis}

The data were analyzed using adequate statistical tests with the aim of looking for agreement between the two diagnostic methods. In case of disagreement, ECGs were reevaluated by a data committee consisting of three ECG experts, who then provided a final report.

Continuous variables were expressed as mean \pm standard deviation, and categorical variables were stored as number of cases or percentage. The Student t-test was used for comparison of continuous variables, while categorical variables were compared using the $\chi^{2}$ test or the Fisher exact test, as appropriate among the groups. All tests were two-tailed and a p-value of $<0.05$ was considered significant. All statistical analyses were performed using MedCalc 17.6 software (MedCalc Software, Ostend, Belgium).

\section{Results}

A total of $99 \mathrm{KM} \mathrm{ECGs} \mathrm{and} 100$ 12-lead ECGs were analyzed. In 1 patient, KM recording was not possible due to an underlying condition (tremors secondary to Parkinson's disease). Comparison of KM ECGs and 12-lead ECGs revealed that the overall quality of the latter was superior (6 vs. 0 - poor, 41 vs. 1 - acceptable, 52 vs. 99 - good, $\mathrm{p}<0.001)$. Effectiveness evaluation of KM with regards to rhythm determination was performed by comparing it with 12-lead ECG interpretation. Sensitivity and specificity were tested for the following rhythms:

- sinus rhythm $-98.4 \%$ and $74.2 \%(\mathrm{p}=0.02)$;

- atrial fibrillation $-92.8 \%$ and $100 \%(\mathrm{p}=0.32)$;

- atrial flutter $-100 \%$ and $100 \%$ (p value not applicable);

- pacemaker rhythm $-53.6 \%$ and $100 \%(\mathrm{p}=$ $=0.008$ ).

Sensitivity and specificity of KM for pathological Q wave detection was $20.6 \%$ and $93.7 \%$, respectively ( $\mathrm{p}<0.001)$. Mean $\mathrm{PQ}, \mathrm{RR}$ and QT measurements in KM ECGs and 12-lead ECGs were as follows:

- $\mathrm{PQ}-160 \mathrm{~ms}$ vs. $180 \mathrm{~ms}(\mathrm{p}<0.001)$;

- RR $-853 \mathrm{~ms}$ vs. $880 \mathrm{~ms}(\mathrm{p}=0.03)$;

- QT $-393 \mathrm{~ms}$ vs. $400 \mathrm{~ms}(\mathrm{p}<0.001)$.

\section{Discussion}

\section{Principal results}

With regard to the fact that many life-threatening arrhythmias are non-sustained and occur infrequently, it is justified to search for relatively cheap solutions giving the patient a chance to record an ECG at the onset of unsettling symptoms. Apart from the conventional, clinically-approved methods, recent years have brought a few breakthroughs regarding smartphone-dependent devices enabling such a procedure. Versatility of mobile phones and general access to the Internet has created the possibility to remotely provide the practitioners with information of possible clinical significance, and thus enable them to react in case of an emergency.

Although reliability of such solutions has not yet been thoroughly investigated in randomized clinical trials, some data suggests good compliance and improved patient management. The most obvious application of smartphone-compatible ECG devices that has been brought up is the possibility of screening for patients with paroxysmal AF especially for those who need antithrombotic therapy [2-4].

The present study confirmed that KM, despite its simplicity, provides a record of sufficient quality to diagnose periods of $\mathrm{AF}$. $\mathrm{KM}$ has also proven to be reliable while supervising QTc period in patients on dofetilide [5] and in pediatric patients with various arrhythmic disorders [6]. In contrast study revealed that QT measurements in KM ECGs are shorter on average by an average of $7 \mathrm{~ms}$, whereas $\mathrm{PQ}$ intervals tend to be shorter by as much as $20 \mathrm{~ms}$ (more than a $10 \%$ difference compared to the conventional ECG). This phenomenon has not as yet been described in the literature available and may potentially mimic arrhythmia, e.g. preexcitation syndrome in extreme cases or conceal atrioventricular blocks, but this tendency was not confirmed in this study.

Extremely high sensitivity compared to relatively lower specificity of KM detection of sinus rhythm is a characteristic that potentially was previously heralded by Narasimha et al. [7] who reported that nearly one third of patients declaring alarming symptoms while the ECG was assessed normal by the algorithm. One in four patients in our population ascribed by KM to sinus rhythm category should in fact have been described differently. Based on the construction of the KM device, it is not surprising that it has low sensitivity in detecting pacemaker rhythm, this supports Desteghe et al. [8] findings and recommendations. 
According to available research, this is the first study to investigate and compare selected parameters in KM records and conventional 12-lead ECGs. New studies, currently with protocols available $[9,10]$ will shortly verify the usefulness of the method and the present findings.

New studies being currently conducted can be also groundbreaking in respect to ischemia detection, and thus rapid detection of myocardial infarction in symptomatic patients $[11,12]$.

\section{Limitations of the study}

Despite the prospective design of this study there are some important limitations. Firstly, this is a single center study. Secondly, ECG interpretation is subjective and thus there may have been certain discrepancies. There were only three teams analyzing the records and assessments of ECG quality were subjective according to their expertise which may somehow bias this study. Furthermore, a group of stable subjects were analyzed, which made it impossible to verify KM applicability for cases of acute cardiac events. Finally, the number of patients included in the study is relatively small and a larger group would enable more reliable conclusions.

\section{Comparison with prior work}

The onset of certain clinical symptoms such as syncope, palpitations and chest pain may indicate an underlying cardiovascular etiology. Among others, paroxysmal arrhythmias are a potential culprit. Long-term ECG monitoring has proven to be an important measure in diagnosing elusive periods of AF, supraventricular arrhythmias and ventricular arrhythmias $[4,13,14]$. To confirm the relation between the symptoms and arrhythmias, the European Society of Cardiology (ESC) guidelines recommend continuous or intermittent ambulatory ECG monitoring. This procedure also makes it possible to verify if the arrhythmic events are secondary to an ischemic heart disease [13, 15]. An appropriate, long-term ECG screening may warn the clinician of a series of potential adverse clinical events. Sudden cardiac death (SCD), one of the leading direct causes of mortality in the western world, whether secondary to ischemic heart disease or to congenital malfunctioning of the electrical conductance system of the heart, could be in many cases prevented by pharmacotherapy or implantable cardioverter-defibrillator (ICD) implantation [13]. Furthermore, paroxysmal AF is a proven risk factor for stroke, another important cause of mortality and disability in developed societies [16-18]. Up until recently it has been estimated that even $20-40 \%$ of ischemic strokes remain idiopathic, but some clinical trials have shown a higher prevalence of paroxysmal AF detectable on a long-term ECG analysis in comparison with a standard $24 \mathrm{~h}$ Holter monitoring in this group of patients [19-22]. The KM system was assessed in a study conducted by Halcox et al. [22] in a randomized control trial (REHEARSE-AF study) in a group of patients older than 65 years with an elevated risk of cerebral thromboembolism $\left(\mathrm{CHA}_{2} \mathrm{DS}_{2}\right.$-VASc score $\left.\geq 2\right)$. The results presented a proactive approach of regular weekly iECG monitoring with the use of KM system may not only be preventive in a medical sense, but also cost-effective in AF detection. This observation opens the discussion over the possibility of a more routine implementation of KM in clinical practice to remotely monitor QT periods.

Rapid diagnosis of AF can prevent cerebral thromboembolic events, and according to recent publication by Rattanawong et al. [23], it can also reduce overall SCD rate. As AF has been reported to provide higher risk of SCD than other associated factors, such as previous myocardial infarction, heart failure or coronary artery disease, preventive measures using quick and easy diagnostic tools such as KM could be undertaken to avoid unnecessary hospitalizations and reduce mortality in this subgroup.

Another important feature of every telemonitoring device that needs to be discussed is its availability. Surveys gathered from subjects enrolled in the SPEAR trial showed a high level of satisfaction and willingness to continue implementing KM ECG testing in the future [4]. Furthermore, all the patients that took part in the present study showed a positive attitude towards KM as well. Such attitudes have also been observed in a recent study by Halcox et al. [22] with patients of advanced age being enthusiastic about such methods.

Patients can be subjected to more personalized care due to the possibility of remote transmission of their ECG records to their physician. In recent years a proactive attitude was positively tested by Klein-Wiele et al. [24], who provided data of successful cooperation between patients, general practitioners and cardiologists. The KM device could be part of a cross-sector telemetric network with rapid response to a patients' worsening state of health by qualified professionals.

Apart from clinical reliability, it also needs to be verified whether smartphone-based ECG controls are not an excessive financial burden for the 
health care system. Initial data suggest that there is no long-term difference in cost-efficiency between conventional monitoring and smartphone supervision, while patient self-awareness seems to benefit from remote self-controls [25]. Nevertheless, this is still a matter of debate as the information at hand are scarce.

Kardia Mobile aside, smartphone-based ECG device market constantly expands, offering new solutions for mobile phones with Android operating systems [26], or enabling a context-aware ECG monitoring [27]. Moreover, specifically oriented systems such as Remote Cardio Control (RCC) designed to diagnose signs of myocardial infarction, continue to be created [28]. It can be conceived that in the following years devices encompassing those standards of diagnostic procedures will become a part of routine approach.

It should be also noted that the number of reports considering mobile ECG record is rising. Apart from arrhythmias considered in this study, there is a possibility to record and diagnose other heart rhythm disturbances, including atrioventricular block [29]. This poses even greater area for implementation of these devices into general practitioner practice.

\section{Conclusions}

The usefulness of $\mathrm{KM}$ is unquestionable in the prevention and rapid detection of $\mathrm{AF}$ and AFls in a standard comparable to the conventional method, namely 12-lead ECG. Applying this novel device to routine diagnostics is rather preemptive, as discrepancies in sensitivity of the algorithm may present a possible misinterpretation of the outcome. The clinical need for an easy-to-use mobile ECG is immense, however it is concluded herein, that further studies are needed to confirm diagnostic value.

\section{Acknowledgements}

The research was performed within the statutory fund of the First Chair and Department of Cardiology of the Medical University of Warsaw and received no external funding.

The authors would like to acknowledge the 9 physicians who assessed ECG recordings during the study and the technicians for recording the ECGs and thus, for making the study possible. Many thanks to the participants for their involvement in the study.
Conflict of interest: Lukasz Koltowski and Paweł Balsam are consultants to Medtronic, and Abbott. Lukasz Koltowski is a consultant to Boston Scientific, and Shareholder of SmartMedics Ltd.

\section{References}

1. Baranowski R, Bieganowska K, Kozłowski D, et al. Zalecenia dotyczące stosowania rozpoznań elektrokardiograficznych. Kardiol Pol. 2010; 68: 335-390.

2. Olgun Kucuk H, Kucuk U, Yalcin M, et al. Time to use mobile health devices to diagnose paroxysmal atrial fibrillation. Int J Cardiol. 2016; 222: 1061, doi:10.1016/j.ijcard.2015.10.159, indexed in Pubmed: 26522994.

3. Tarakji KG, Wazni OM, Callahan T, et al. Using a novel wireless system for monitoring patients after the atrial fibrillation ablation procedure: the iTransmit study. Heart Rhythm. 2015; 12(3): 554-559, doi: 10.1016/j.hrthm.2014.11.015, indexed in Pubmed: 25460854.

4. Pruszczyk P, Tomaszuk-Kazberuk A, Słowik A, et al. Management of bleeding or urgent interventions in patients treated with direct oral anticoagulants: 2017 recommendations for Poland. Pol Arch Intern Med. 2017; 127(5): 343-351, doi: 10.20452/ pamw.3995, indexed in Pubmed:28400546.

5. Chung EH, Guise KD. QTC intervals can be assessed with the AliveCor heart monitor in patients on dofetilide for atrial fibrillation. J Electrocardiol. 2015; 48(1): 8-9, doi: 10.1016/j.jelectrocard.2014.10.005, indexed in Pubmed: 25453194.

6. Nguyen HH, Van Hare GF, Rudokas M, et al. SPEAR Trial: Smartphone Pediatric ElectrocARdiogram Trial. PLoS One. 2015; 10(8): e0136256, doi:10.1371/journal.pone.0136256, indexed in Pubmed: 26295569.

7. Narasimha D, Hanna N, Beck H, et al. Validation of a smartphone-based event recorder for arrhythmia detection. Pacing Clin Electrophysiol. 2018; 41(5): 487-494, doi: 10.1111/ pace.13317, indexed in Pubmed: 29493801.

8. Desteghe L, Raymaekers Z, Lutin M, et al. Performance of handheld electrocardiogram devices to detect atrial fibrillation in a cardiology and geriatric ward setting. Europace. 2017; 19(1): 29-39, doi: 10.1093/europace/euw025, indexed in Pubmed: 26893496.

9. Guhl EN, Schlusser CL, Henault LE, et al. Rationale and design of the Atrial Fibrillation health Literacy Information Technology Trial: (AF-LITT). Contemp Clin Trials. 2017; 62: 153-158, doi: 10.1016/j.cct.2017.09.005, indexed in Pubmed: 28923492.

10. Treskes RW, Gielen W, Wermer MJ, et al. Mobile phones in cryptogenic strOke patients Bringing sIngle Lead ECGs for Atrial Fibrillation detection (MOBILE-AF): study protocol for a randomised controlled trial. Trials. 2017; 18(1): 402, doi: 10.1186/ s13063-017-2131-0, indexed in Pubmed:28851409.

11. Barbagelata A, Bethea CF, Severance HW, et al. Smartphone ECG for evaluation of ST-segment elevation myocardial infarction (STEMI): Design of the ST LEUIS International Multicenter Study. J Electrocardiol. 2018; 51(2): 260-264, doi: 10.1016/j.jelectrocard.2017.10.011, indexed in Pubmed:29174099.

12. Muhlestein JB, Le V, Albert D, et al. Smartphone ECG for evaluation of STEMI: results of the ST LEUIS Pilot Study. 
J Electrocardiol. 2015; 48(2): 249-259, doi: 10.1016/j.jelectrocard.2014.11.005, indexed in Pubmed: 25601407.

13. Priori SG, Blomström-Lundqvist C, Mazzanti A, et al. 2015 ESC Guidelines for the management of patients with ventricular arrhythmias and the prevention of sudden cardiac death: The Task Force for the Management of Patients with Ventricular Arrhythmias and the Prevention of Sudden Cardiac Death of the European Society of Cardiology (ESC). Endorsed by: Association for European Paediatric and Congenital Cardiology (AEPC). Eur Heart J. 2015; 36(41): 2793-2867, doi: 10.1093/eurheartj/ehv316, indexed in Pubmed: 26320108.

14. Solomon MD, Yang J, Sung SH, et al. Incidence and timing of potentially high-risk arrhythmias detected through long term continuous ambulatory electrocardiographic monitoring. BMC Cardiovasc Disord. 2016; 16: 35, doi: 10.1186/s12872-016-0210-x, indexed in Pubmed: 26883019.

15. Roffi M, Patrono C, Collet JP, et al. 2015 ESC Guidelines for the management of acute coronary syndromes in patients presenting without persistent ST-segment elevation: Task Force for the Management of Acute Coronary Syndromes in Patients Presenting without Persistent ST-Segment Elevation of the European Society of Cardiology (ESC). Eur Heart J. 2016; 37(3): 267-315, doi: 10.1093/eurheartj/ehv320, indexed in Pubmed: 26320110.

16. Wolf PA, Abbott RD, Kannel WB. Atrial fibrillation as an independent risk factor for stroke: the Framingham Study. Stroke. 1991; 22(8): 983-988, indexed in Pubmed: 1866765.

17. Camm AJ, Lip GYH, De Caterina R, et al. 2012 focused update of the ESC Guidelines for the management of atrial fibrillation: an update of the 2010 ESC Guidelines for the management of atrial fibrillation. Developed with the special contribution of the European Heart Rhythm Association. Eur Heart J. 2012; 33(21): 2719-2747, doi: 10.1093/eurheartj/ehs253, indexed in Pubmed: 22922413.

18. Michniewicz E, Mlodawska E, Lopatowska P, et al. Patients with atrial fibrillation and coronary artery disease: Double trouble. Adv Med Sci. 2018; 63(1): 30-35, doi: 10.1016/j.advms.2017.06.005, indexed in Pubmed: 28818746.

19. Sanna T, Diener HC, Passman RS, et al. CRYSTAL AF Investigators. Cryptogenic stroke and underlying atrial fibrillation. $\mathrm{N} \mathrm{Engl}$ J Med. 2014; 370(26): 2478-2486, doi: 10.1056/NEJMoa1313600, indexed in Pubmed: 24963567.
20. Gladstone DJ, Dorian P, Spring M, et al. Atrial fibrillation in patients with cryptogenic stroke. N Engl J Med. 2014; 370(26): 2467-2477, doi:10.1056/NEJMoa1311376, indexed in Pubmed: 24963566.

21. Lilli A, Di Cori A. The cold facts of long-term ECG monitoring. Expert Rev Cardiovasc Ther. 2015; 13(2): 125-127, doi: 10.1586 /14779072.2015.998201, indexed in Pubmed: 25555394.

22. Halcox JPJ, Wareham K, Cardew A, et al. Assessment of Remote Heart Rhythm Sampling Using the AliveCor Heart Monitor to Screen for Atrial Fibrillation: The REHEARSE-AF Study. Circulation. 2017; 136(19): 1784-1794, doi: 10.1161/CIRCULATIONAHA.117.030583, indexed in Pubmed:28851729.

23. Rattanawong P, Upala S, Riangwiwat T, et al. Atrial fibrillation is associated with sudden cardiac death: a systematic review and meta-analysis. J Interv Card Electrophysiol. 2018; 51(2): 91-104, doi: 10.1007/s10840-017-0308-9, indexed in Pubmed: 29332241.

24. Klein-Wiele O, Faghih M, Dreesen S, et al. A novel cross-sector telemedical approach to detect arrhythmia in primary care patients with palpitations using a patient-activated event recorder. Cardiol J. 2016; 23(4): 422-428, doi: 10.5603/CJ.a2016.0033, indexed in Pubmed: 27320955.

25. Bloss CS, Wineinger NE, Peters M, et al. A prospective randomized trial examining health care utilization in individuals using multiple smartphone-enabled biosensors. PeerJ. 2016; 4: e1554, doi: 10.7717/peerj.1554, indexed in Pubmed: 26788432.

26. Choo KY, Ling HC, Lo YC, et al. Android based self-diagnostic electrocardiogram system for mobile healthcare. Technol Health Care. 2015; 23 Suppl 2: S435-S442, doi: 10.3233/THC-150980, indexed in Pubmed: 26410510.

27. Miao F, Cheng Y, He Yi, et al. A wearable context-aware ECG monitoring system integrated with built-in kinematic sensors of the smartphone. Sensors (Basel). 2015; 15(5): 11465-11484, doi: 10.3390/s150511465, indexed in Pubmed: 25996508.

28. Cinaglia P, Tradigo G, Guzzi PH, et al. Design and Implementation of a Telecardiology System for Mobile Devices. Interdiscip Sci. 2015; 7(3): 266-274, doi: 10.1007/s12539-015-0267-8, indexed in Pubmed: 26223546.

29. Balsam P, Gawalko M, Lodziński P, et al. Atrioventricular block registration with smart phone associated ECG device. Heart Beat J. 2017; 1: 54-55, doi:10.24255/hbj/68109. 